\title{
El trasplante intracoronario de células mononucleares de médula ósea se asocia a mejoría la función ventricular izquierda, en pacientes con cardiopatía dilatada de diferente etiología. Proyecto INNOVA Chile N²05-4350.
}

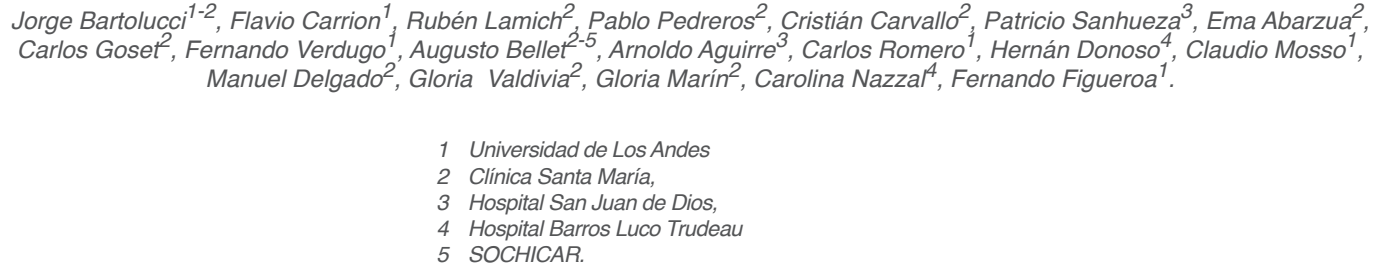

Introducción: Estudios recientes indican que el trasplante intracoronario de células mononucleares de médula ósea (BMCs) autólogas, mejoran la fracción de eyección (FEVI) y otros marcadores clínicos en pacientes con insuficiencia cardíaca (IC).

Objetivo: Evaluar la seguridad y eficacia de la administración intracoronaria de BMCs autólogas, en pacientes con insuficiencia cardíaca (IC) en fase dilatada, de diferente etiología y en óptimas condiciones de tratamiento médico.

Método: De 23 pacientes consecutivos que cumplieron con los criterios de inclusión, 12 fueron asignados a trasplante intracoronario de BMCs autólogas, recibiendo una dosis media de $8.19 \pm 4.43 \times 10^{6}$ células CD34+ (Grupo trasplantado). Los pacientes restantes sólo recibieron terapia estándar (Grupo control). Todos los pacientes fueron evaluados mediante Electrocardiograma, Ecocardiografía, Holter ECG, RMN Cardíaca, Test de esfuerzo, Potenciales Ventriculares Tardíos, Variabilidad de Frecuencia Cardíaca y evaluación clínica a los 0, 3,6 y 12 meses. La capacidad funcional (CF) fue evaluada clínicamente y por cuestionarios de calidad de vida. El análisis estadístico fue realizado mediante Test Anova, y test de Bonferroni.

Resultados: El grupo trasplantado presentó un aumento significativo de la FEVI a los 6 meses $(26.75 \pm 4.85$ vs $37.82 \pm 6.97 \%, \mathrm{p}=0.001$ ) mejoría que se mantuvo a los 12 meses ( $26.75 \pm 4.85$ vs $37.27 \pm 7.51 \%, \mathrm{p}=0.002)$. Hubo una mejora significativa de la $\mathrm{CF}$ en el grupo trasplantado a los 6 y 12 meses $(p<0.001)$. No hubo cambios significativos en los volúmenes de ventrículo izquierdo, así como en las restantes variables estudiadas. En el grupo control no observamos cambios de estas variables. No hubo complicaciones en relación al trasplante de BMCs. Conclusión: En pacientes con IC severa y baja FEVI, el trasplante intracoronario de células BMCs autólogas, se asoció a una mejoría significativa de la FEVI y la CF, a los 6 y 12 meses. Adicionalmente, no observamos complicaciones en relación con el procedimiento, lo que confirma la seguridad del trasplante intracoronario de BMCs.

Palabras claves: Trasplante de células de médula ósea, Función ventricular, Insuficiencia cardíaca. 


\section{Autologous bone marrow cell transplantation improves Ejection Fraction in Patients with Dilated Cardiomyopathy of Diverse etiologies. Proyecto INNOVA Chile N²05-4350.}

Background: Recent studies indicate that intracoronary delivery of autologous bone marrow mononuclear cells (BMCs) improves the ejection fraction (LVEF) and other clinical markers in patients with heart failure (HF).

Aim: To evaluate the safety and efficacy of intracoronary delivery of autologous BMCs in patients with $\mathrm{HF}$ in dilated phase under optimal medical treatment. Method: Of 23 consecutive patients who met the inclusion criteria, 12 were assigned to autologous BMCs intracoronary transplantation, receiving a mean dose of $8.19 \pm 4.43 \times 106$ CD34+ cells (BMCs group). The remaining patients received only standard therapy (control group). All patients were evaluated by Electrocardiogram, Echocardiography, Holter Monitoring, Cardiac Magnetic Resonance Imaging, Stress Testing, Ventricular Late potetials, Heart Rate Variability, and regular clinical examination at baseline and at follow-up (3, 6 and 12 months). Repeated measures ANOVA and Bonferroni testing were used

\section{Introducción}

Durante las dos últimas décadas, hemos sido testigos de la disminución de la mortalidad precoz en los pacientes post infarto agudo al miocardio (IAM). La situación ha sido diferente en los pacientes con cardiopatía coronaria crónica, observándose un incremento en las consultas médicas y hospitalizaciones debido a insuficiencia cardíaca (IC), como consecuencia de la disfunción ventricular izquierda. Esto acontece a pesar del enorme desarrollo de la terapia farmacológica, la masificación de la angioplastía primaria y la incorporación de nuevos dispositivos. Este importante arsenal terapéutico disponible, no ha logrado revertir en su totalidad el daño miocárdico producto de la necrosis celular, la que conduce a la pérdida de la función contráctil, a una progresiva dilatación e IC ${ }^{\mathbf{1}}$.

La situación antes planteada ha obligado a la búsqueda de enfoques terapéuticos adicionales, que permitan optimizar la inadecuada capacidad reparativa endógena del corazón, producto de la injuria por isquemia $u$ otras causas, dirigiendo la mirada hacia la Medicina Regenerativa, utilizan- for statistic analysis.

Results: The BMCs group presented a significant increase in EF at sixth months $(26.75 \pm 4.85$ vs. $37.82 \pm 6.97 \%, \mathrm{p}=0.001)$ and 12 months posttransplant ( $26.75 \pm 4.85$ vs. $37.27 \pm 7.51 \%$, $\mathrm{p}=0.002)$. There was a significant improvement in functional (NYHA) in the transplanted group at 6 and 12 months $(p<0.001)$. There were no significant changes concerning left ventricular volumes, heart rate variability and exercise stress testing. We observed no improvement of these variables in the control group. There were no complications related to the BMCs transplant.

Conclusions: Intracoronary infusion of autologous BMCs, in addition to standard therapy, was associated with significant improvement of left ventricular function at 12 months in patients with HF. We observed no complications relative to the procedure.

Keywords: Bone marrow cell transplantation, Left ventricular function, Heart failure

do para ello células madre o progenitoras, que conduzcan a una verdadera reparación del daño miocardio y, tal vez, a su regeneración ${ }^{23}$. Durante los últimos años la mayor parte de las investigaciones de orden clínico, han utilizado con mayor frecuencia células madre autólogas ${ }^{4,5}$ y ocacionalmente alogénicas ${ }^{6,7}$, de diferentes fuentes celulares, como mononucleares de médula ósea y sangre periférica, mioblastos de músculo esquelético y mesenquimales de médula ósea, siendo las vías de implantación también variadas (intracoronaria, transendocárdica, transpericárdica, endovenosa y pericárdica) ${ }^{2}$. Son numerosos los estudios experimentales $^{8-14}$ y clínicos ${ }^{15-22}$ que han evaluado el tratamiento intracoronario con células madre alogénicas, con mayor frecuencia mononucleares de médula ósea (BMCs), en pacientes post infarto agudo al miocardio con posterioridad al uso de la angioplastía primaria y con menos frecuencia en la enfermedad coronaria crónica ${ }^{23-26}$ y las miocardiopatías. ${ }^{27,28}$ Las perspectivas son promisorias, documentando una mejoría de la fracción de eyección de ventrículo izquierdo (FEVI) y de otros marcadores clínicos post implante. Estudios controlados recientes han 
objetivado una disminución del potencial arritmogénico y reducción de la mortalidad alejada, ${ }^{\mathbf{2 1}, 25}$ Dos metaanálisis recientes han sugerido que el tratamiento con BMCs en pacientes post IAM podría conducir en el largo plazo a una mejoría de la función ventricular izquierda, adicional a las terapias convencionales y la angioplastía primaria (Cochrane Library el 2012) ${ }^{\mathbf{4}, 5}$. Aunque ambos metaanálisis parecen ser optimistas en relación con la seguridad y eficacia de las células madre en el tratamiento del IAM, quedan muchas interrogantes por resolver. Sin embargo, los resultados documentados en dos publicaciones recientes incluidas en este análisis, son muy importantes, ya que evidencian una mejoría en la sobrevida (Grajek 2010, Assmus 2010). ${ }^{29,30}$ Este hecho debe ser necesariamente confirmado en ensayos clínicos en fase tres. En el metaanálisis de la Universidad de Kansas se señaló adicionalmente una disminución en la mortalidad, la recurrencia de IAM y de la trombosis del stent ${ }^{5}$. En este momento hay alrededor de 33 estudios en marcha que podrán precisar con mayor certeza estos hallazgos.

Objetivo del Estudio

Nuestro objetivo primario estuvo orientado a evaluar la seguridad del implante intracoronario de las células BMCs en un grupo de pacientes crónicos con cardiopatía en fase dilatada e IC, en óptimas condiciones de tratamiento médico.

El objetivo secundario estuvo dirigido a demostrar eficacia de la terapia empleada, documentada a través de parámetros de función ventricular y de capacidad funcional.

\section{Métodos}

Se trata de un estudio en fase I-II, prospectivo, controlado y ciego para los evaluadores de los procedimientos técnicos.

\section{Características de la población estudiada}

Se seleccionó un total de 23 pacientes consecutivos en IC crónica en fase dilatada, que cumplieron con los criterios de inclusión, fueron derivados a Clínica Santa María desde dos hospitales públicos y desde la misma institución. No hubo una selección por patología específica, con la finalidad de ver la respuesta de esta terapia en una población de pacientes de consulta frecuente. Los 23 pacientes fueron ingresando al estudio en forma progresiva, los 12 primeros fueron seleccionados para ser trasplantados con MBCs, siendo los 11 restantes asignados al grupo control. Criterios de inclusión: Insuficiencia cardíaca estable sintomática y en óptimas condiciones respecto a su tratamiento estándar, fracción de eyección igual o inferior a un 40\%, edad entre 18 y 75 años, arterias coronarias abiertas en el caso de pacientes coronarios y normales o con lesiones no significativas en los no coronarios, consentimiento informado aceptado por cada paciente, aprobación previa del protocolo por parte de los comités de ética de los hospitales y Servicios de Salud respectivos.

Criterios de exclusión: Insuficiencia cardíaca severa y persistente, taquicardia ventricular no controlada, enfermedad maligna concomitante, enfermedad coronaria con isquemia no resuelta, síndrome coronario agudo inferior a 12 meses, fibrilación auricular crónica con frecuencia ventricular no controlada, insuficiencia renal, infección activa, enfermedad hematológica, consumo de alcohol en forma exagerada y/o drogas los últimos 12 meses a la incorporación al estudio.

\section{Métodos de evaluación}

Los pacientes fueron evaluados al ingresar al protocolo con los siguientes exámenes: electrocardiograma, test de esfuerzo de acuerdo a protocolo de Naughton, ecocardiograma doppler color con strain rate, holter para la evaluación de arritmias, potenciales ventriculares tardíos en la búsqueda de potencial arritmogénico (criterios de positividad de acuerdo a la ESC y AHA), variabilidad de la frecuencia cardíaca en dominio del tiempo, resonancia magnética nuclear y laboratorio clínico (hemograma, recuento plaquetario, creatininemia, glicemia, orina, electrolitos plasmáticos, CK total, $\mathrm{CK} \mathrm{MB}$, y troponina). Estos exámenes se repitieron a los 3,6 y 12 meses de seguimiento. Los ecocardiogramas fueron realizados en un ecocardiógrafo General Electric Vivid 7 Dimension, la FEVI fue medida por Simpson modificado, calculando los volúmenes de final de diástole y final de sístole, en visión apical de dos y cuatro cámaras. Las mediciones de las cavidades y el estudio de la función diastólica se realizaron de acuerdo a las guías de las sociedades de ecocardiografía americana y europea.

La evaluación de la capacidad funcional (CF) se efectuó en base a la clasificación de la NYHA. También se realizó una valoración de la CF en forma retrospectiva, a través de dos cuestionarios de calidad de vida para insuficiencia cardíaca; el de Minnesota Living with Heart Failure Questionnaire (MLHFQ) y el de miocardiopatía de la ciudad de Kansas, City Cardiomyophaty Questionnaire (KCCQ).

\section{Metodología obtención, procesamiento y bioseguridad de las BMCs:}

Las BMCs autólogas fueron obtenidas por punción bilateral de la cresta iliaca, efectuada simultáneamente por 
dos hematólogos, aspirando un volumen promedio de $210 \pm 10.87 \mathrm{cc}$, de este volumen, se envió 1cc al laboratorio de bacteriología y el restante a nuestro laboratorio GMP (Good Manufacturing Practices), donde las células mononucleares fueron separadas mediante gradiente de densidad por medio de Ficoll-histopaque (Amersham, GE(C). Posteriormente, se determinó la viabilidad del producto celular y se realizó marcación con anticuerpos anti CD34 y CD45 o con control de isotipo, IsoClonic ControlPE (Beckmann Coulter, USA). El porcentaje de células CD34+ fue determinado por citometría de flujo en Coulter Epics XL ${ }^{\mathrm{TM}}$ (Beckmann Coulter, USA).

La determinación de bioseguridad biológica de la muestra con posterioridad a la separación de las BMCs y previo al implante, se determinó de un compuesto de 500ul de la muestra y de 100 ul de producto celular, los que fueron sometidas a test de hemocultivo Bactalert ${ }^{\circledR}$ (BioMérieux, Fr), resultando negativas en todos los casos.

Para su implantación las BMCs fueron suspendidas en plasma autólogo y transportadas en un tubo estéril, con un volumen de 9-10 cc al servicio de hemodinamia para ser implantadas. El tiempo promediado desde el término del proceso en el laboratorio de terapia celular y la entrega al servicio de hemodinamia fue de 90 minutos.

Los pacientes no recibieron estimulación previa y ninguna fracción de las células fue cultivada.

\section{Trasplante de las BMCs:}

Doce pacientes consecutivos fueron trasplantados con BMCs vía catéter balón, posicionado en arteria coronaria descendente anterior y luego en la arteria coronaria derecha, el catéter fue inflado por 2-3 minutos para detener transitoriamente el flujo coronario anterógrado, permitiendo la anidación del producto celular y su difusión a nivel microvascular, de acuerdo con técnica previamente descrita19. El volumen total inyectado fue de 9-10 cc, la mitad en el territorio coronario izquierdo y la otra en el derecho. La dosis total de BMCs implantadas fue de 1.94 x $108 \pm 5.3$ x 107, correspondiendo a un promedio de células CD 34+ por paciente de 8.19 $44.43 \times 106$.

Con posterioridad al procedimiento los pacientes fueron hospitalizados en una unidad de intermedio coronario por 24 horas, donde se mantuvieron monitorizados. A todos se les efectuó electrocardiograma previo y posterior al procedimiento, así como medición de $\mathrm{CK}$ total, $\mathrm{CKmb}$, troponinas y función renal. Al día siguiente, el paciente era dado de alta con su tratamiento médico habitual, controlado telefónicamente durante los primeros días por enfermera y médico, en el caso que se estimara necesario. Un control por cardiólogo fue realizado a los 30 días del alta.

\section{Seguimiento:}

Los pacientes tuvieron un seguimiento cardiológico estricto, con una frecuencia de 3, 6 y 12 meses, bajo óptimas condiciones de tratamiento médico (dosis máximas tolerables de diuréticos, IECA, ARA II y beta bloqueadores). Siendo definidos como estables por lo menos 3 meses antes de ser incorporados al estudio. Los exámenes antes descritos se repitieron en cada uno de los controles. La evaluación de la capacidad funcional (CF) se efectuó al ingreso, seis y doce meses, en base a la clasificación de la NYHA, al igual que los cuestionarios de calidad de vida antes descritos.

Todas las ecocardiografías fueron realizadas por dos ecocardiografistas experimentados, sus resultados fueron nuevamente analizados y consensuados al término del estudio. Ninguno de ellos conocía si el paciente había sido tratado con BMCs, de manera de no influir en los datos obtenidos. De igual manera ocurrió con los restantes exámenes cardiológicos no invasivos y la coronariografía previa. Solo el médico que realizó el trasplante intracoronario de BMCs conocía esta información, al igual que el director del proyecto y la enfermera coordinadora.

\section{Análisis Estadístico}

Las variables continuas se expresaron como promedio \pm error estándar de la media (SEM) y las variables categóricas como valor absoluto. Se evaluó la normalidad de las variables continuas mediante el test de Shapiro-Wilk y en consecuencia, aquellas variables que resultaron tener distribución normal fueron comparadas mediante la prueba t-Student para muestras independientes; en caso de descartarse la normalidad las variables fueron comparadas mediante la prueba de Mann-Whitney. Las variables categóricas fueron comparadas usando test de Chi-cuadrado. Para comparaciones de evaluaciones post tratamiento versus situación basal se realizó análisis de la varianza (ANOVA) con análisis post-hoc de Bonferroni. Se consideraron estadísticamente significativas las diferencias con un valor $\mathrm{p}<0.05$. Todos los análisis estadísticos fueron realizados en IBM SPSS Statistics versión 19.

\section{Resultados}

No se observaron complicaciones relativas al trasplante de células BMCs intracoronario, durante su aplicación, ni en ningún momento del seguimiento inmediato. La dosis promedio de células CD34+ implantadas fue de $8.19 \pm 4.43$ x 106. Tampoco observamos com- 
plicaciones relativas a la extracción de médula ósea, a pesar del elevado volumen promedio obtenido por paciente $(200 \pm 10 \mathrm{cc})$.

Un paciente de cada grupo falleció, ambos en forma súbita. El paciente del grupo control falleció a los 17 días de ingresado al protocolo. El paciente del grupo trasplantado falleció a los 2 meses 27 días de ingresado al protocolo. Los dos pacientes alcanzaron a completar su evaluación inicial, en ella se pudo documentar que ambos tenían arritmias ventriculares complejas en el Holter, caracterizadas por extrasístoles ventriculares aisladas frecuentes y escasos colgajos de taquicardia ventricular monomórfica no sostenida.

\begin{tabular}{|l|c|l|l|}
\hline \multicolumn{3}{|c|}{ Tabla 1. Características de la Población Estudiada. } \\
\hline Variables & $\begin{array}{c}\text { Grupo } \\
\text { Tratado (N²) }\end{array}$ & $\begin{array}{l}\text { Grupo } \\
\text { Control (N¹1) }\end{array}$ & P \\
\hline Edad & $57.9 \pm 14$ & $57 \pm 11$ & $\mathrm{~ns}$ \\
Índice masa corporal & $28.2 \pm 4.86$ & $28.6 \pm 4.76$ & $\mathrm{~ns}$ \\
Sexo (género masculino \%) & 66.6 & 81.8 & $\mathrm{~ns}$ \\
Hipertensión & 4 & 4 & $\mathrm{~ns}$ \\
Dislipidemia & 1 & 3 & $\mathrm{~ns}$ \\
Diabetes Mellitus & 2 & 1 & $\mathrm{~ns}$ \\
Tabaco & 3 & 4 & $\mathrm{~ns}$ \\
Alcohol & 2 & 1 & $\mathrm{~ns}$ \\
Cocaína & 1 & - & $\mathrm{ns}$ \\
Quimioterapia & 1 & & \\
& & &
\end{tabular}

Características de la Población Estudiada.

\begin{tabular}{|l|c|c|l|}
\hline \multicolumn{4}{|c|}{ Tabla 2. Características de la Cardiopatía. } \\
\hline Características & $\begin{array}{c}\text { Grupo } \\
\text { Tratado (N'12) }\end{array}$ & $\begin{array}{c}\text { Grupo } \\
\text { Control (N¹) }\end{array}$ & P \\
\hline Miocardiopatía dilatada idiopática & 8 & 5 & $\mathrm{~ns}$ \\
Infarto al miocardio antiguo & 2 & 3 & $\mathrm{~ns}$ \\
Cardiopatía hipertensiva & 2 & 2 & $\mathrm{~ns}$ \\
Enfermedad coronaria crónica & - & 1 & $\mathrm{~ns}$ \\
Insuficiencia mitral moderada o severa & 8 & 2 & 0.004 \\
Disfunción diastólica grado III & 8 & 2 & 0.004 \\
Bloqueo completo rama izquierda & 3 & 2 & $\mathrm{~ns}$ \\
Bloqueo completo rama derecha & 6 & 2 & $\mathrm{~ns}$ \\
Hemibloqueo izquierdo anterior & 6 & 4 & $\mathrm{~ns}$ \\
Fibrilación auricular & 1 & 2 & $\mathrm{~ns}$ \\
Cardiodesfibrilador & - & 1 & $\mathrm{~ns}$ \\
\hline
\end{tabular}

Características de la Cardiopatía.
Adicionalmente, ambos tenían BCRI con asincrónica septal y FEVI de $20 \%$.

A un paciente del grupo control antes de ser incorporado al protocolo se le implantó un cardiodesfibrilador, no documentándose descargas durante su seguimiento. Las características demográficas de ambos grupos, los factores habituales de riesgo cardiovascular, consumo exagerado de alcohol, uso de cocaína o antecedente de tratamiento con quimioterapia pueden ser vistas en la (Tabla 1).

La etiología de las cardiopatías, la existencia de trastornos de la conducción intraventricular y fibrilación auricular, al igual que el grado de insuficiencia mitral y de disfunción diastólica, se detallan en la (Tabla 2).

Los fármacos utilizados en el tratamiento de las cardiopatías de ambos grupos, se describen en la (Tabla 3).

La asincronía septal se observó en todos los pacientes con bloqueo completo de rama izquierda. Tres pacientes del grupo tratado con BMCs (QRSs: 179-184-200 ms), y dos del grupo control (QRSs: 190-171 ms).

Los datos ecocardiográficos de ambos grupos a los 0, 3, 6 y 12 meses, en relación con la función ventricular izquierda, respecto a la FEVI medida por Simpson, volumen final de diástole (VFD) y volumen final de sístole (VFS), se señalan en la (Tabla 4).

\begin{tabular}{|c|c|c|c|}
\hline Medicamentos & $\begin{array}{l}\text { Grupo Tratado } \\
\text { CD34+ }\left(\mathrm{N}^{\circ} 12\right)\end{array}$ & $\begin{array}{c}\text { Grupo } \\
\text { Control }\left(N^{\circ} 11\right)\end{array}$ & $\mathbf{P}$ \\
\hline $\begin{array}{l}\text { Aspirina } \\
\text { Clopidogrel } \\
\text { Acenocumarol } \\
\text { IECH/ARA II } \\
\beta \text {-bloqueador } \\
\text { Diuréticos } \\
\text { Digitálicos } \\
\text { Vasodilatador } \\
\text { Estatinas } \\
\text { Amiodarona }\end{array}$ & $\begin{array}{l}3 \\
1 \\
8 \\
9 \\
10 \\
11 \\
3 \\
3 \\
4 \\
1\end{array}$ & $\begin{array}{l}7 \\
2 \\
7 \\
9 \\
8 \\
10 \\
3 \\
- \\
4 \\
1\end{array}$ & $\begin{array}{l}\text { ns } \\
\text { ns } \\
\text { ns } \\
\text { ns } \\
\text { ns } \\
\text { ns } \\
\text { ns } \\
\text { ns } \\
\text { ns } \\
\text { ns }\end{array}$ \\
\hline
\end{tabular}

Tratamiento Farmacológico.

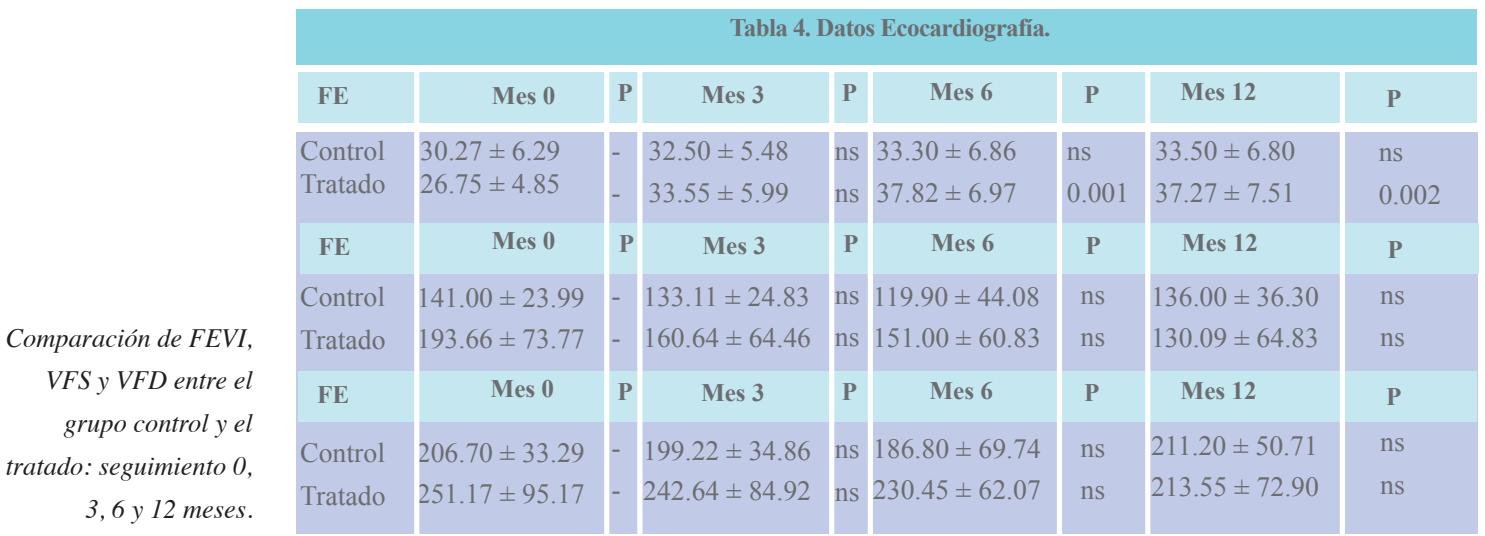


El Holter y el Test de Esfuerzo fue realizado a todos los pacientes, la información entregada por estas técnicas fue semejante para ambos grupos y no aportó datos diferenciadores de interés.

Los datos evolutivos de la CF de acuerdo con la clasificación de la NYHA pueden verse en la (Figura 1). Los datos de calidad de vida ponderados a través de los cuestionarios MLHFQ y KCCQ, se detallan en la (Tabla 5).

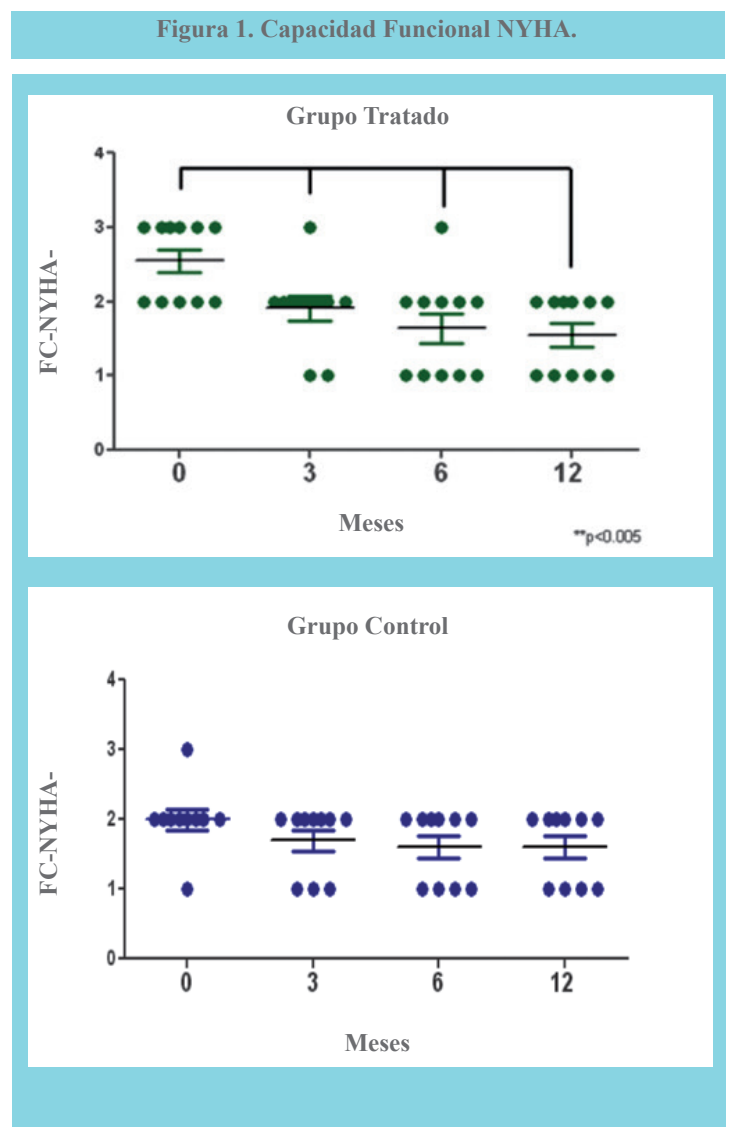

\section{Discusión}

Los dos hechos más relevantes de nuestro trabajo son, por una parte, la necesidad de confirmar en nuestro medio, la seguridad del trasplante de las BMCs intracoronaria en pacientes con cardiopatía e insuficiencia cardíaca crónica y, por otra, la búsqueda de un potencial beneficio de esta terapia, sobre la función ventricular izquierda y la $\mathrm{CF}$.

$\mathrm{Al}$ igual que la mayor parte de los estudios publicados, $, 4,5$ comprobamos que el método es seguro y reproducible. Ninguno de nuestros pacientes trasplantados con BMCs tuvo complicaciones derivadas del procedimiento, ni durante el seguimiento inmediato.

En relación con la eficacia de la terapia empleada, pudimos comprobar al igual que en otros estudios publicados, 4,5 que las BMCs ejercen un efecto beneficioso sobre la función ventricular izquierda, evidenciado de manera más manifiesta en la FEVI. Al momento del ingreso al protocolo, el grupo control y el trasplantado no tenían diferencias significativas respecto a la FEVI, manteniéndose esta situación a los tres meses de seguimiento (Tabla 4). Solo se hizo evidente un cambio manifiesto en la FEVI a los 6 meses ( $\mathrm{p} 0.001$ ), el que se mantuvo a los 12 meses post trasplante (p 0.002).

Estudios recientes con BMCs implantadas de manera intramiocardica, señalan que el proceso de recuperación es más lento, observándose una mejoría más evidente de la función regional del ventrículo izquierdo a partir de los 3 meses. Este hecho predice la detención del remodelado ventricular, apareciendo como consecuencia cambios en la cavidad ventricular, que sólo fueron significativos a los 6 meses $^{\mathbf{2 6}}$. Aunque el procedimiento de implante utilizado y la población estudiada en esta publicación es diferente a la nuestra, de alguna manera explica los resultados, ya que

\begin{tabular}{|c|c|c|c|c|c|c|}
\hline \multirow[b]{2}{*}{ Cuestionarios } & \multicolumn{3}{|c|}{ Grupo Control $\left(n^{\circ}=8\right)$} & \multicolumn{3}{|c|}{ Grupo Tratado $\left(n^{\circ}=11\right)$} \\
\hline & Basal & Actual & $\mathbf{P}$ & Basal & Actual & $\mathbf{P}$ \\
\hline MLHFQ & $54.00 \pm 23.62$ & $36.00 \pm 23.07$ & 0.145 & $54.00 \pm 23.62$ & $33.25 \pm 25.42$ & 0.001 \\
\hline KCCQ & Basal & Actual & $\mathbf{P}$ & Basal & Actual & $\mathbf{P}$ \\
\hline $\begin{array}{l}\text { Limitación Funcional } \\
\text { Síntomas } \\
\text { Calidad de vida } \\
\text { Interferencia social } \\
\text { Autoeficacia } \\
\text { Estado funcional } \\
\text { Resumen clínico }\end{array}$ & $\begin{array}{l}0.625 \pm 0.351 \\
0.475 \pm 0.283 \\
0.523 \pm 0.281 \\
0.766 \pm 0.279 \\
0.521 \pm 0.198 \\
0.538 \pm 0.271 \\
0.551 \pm 0.254\end{array}$ & $\begin{array}{l}0.708 \pm 0.290 \\
0.734 \pm 0.190 \\
0.656 \pm 0.281 \\
0.844 \pm 0.291 \\
0.719 \pm 0.178 \\
0.725 \pm 0.207 \\
0.723 \pm 0.215\end{array}$ & $\begin{array}{l}0.522 \\
0.048 \\
0.365 \\
0.651 \\
0.053 \\
0.148 \\
0.185\end{array}$ & $\begin{array}{l}0.326 \pm 0.250 \\
0.309 \pm 0.256 \\
0.244 \pm 0.304 \\
0.375 \pm 0.349 \\
0.500 \pm 0.220 \\
0.325 \pm 0.251 \\
0.314 \pm 0.263\end{array}$ & $\begin{array}{l}0.845 \pm 0.276 \\
0.811 \pm 0.228 \\
0.778 \pm 0.303 \\
0.966 \pm 0.525 \\
0.795 \pm 0.172 \\
0.832 \pm 0.238 \\
0.818 \pm 0.253\end{array}$ & $\begin{array}{l}0.002 \\
0.001 \\
0.003 \\
0.004 \\
0.002 \\
0.001 \\
0.001\end{array}$ \\
\hline
\end{tabular}

Cuestionario Calidad de Vida con Insuficiencia Cardiaca de Minnesota (MLHFQ).

Cuestionario de Cardiomiopatía de Kansas City (KCCQ). 
el tiempo necesario para detener el proceso de remodelado ventricular, requiere de un plazo más prolongado.
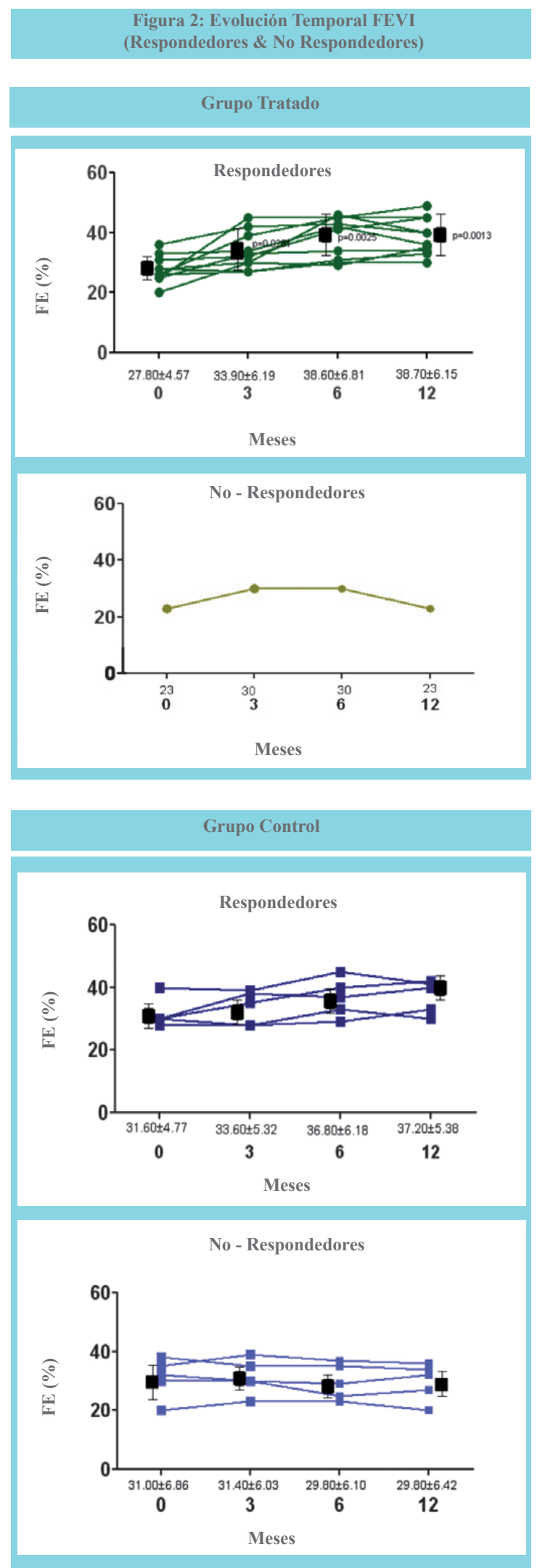

A diferencia de otros autores ${ }^{\mathbf{5}}$ no observamos entre ambos grupos diferencias significativas respecto al volumen final de diástole (VFD). El volumen final de sístole (VFS) tampoco mostró diferencias significativas entre ambos grupos, sin embargo, a los 12 meses se documentó un descenso evidente de este $(193.6 \pm 73.7 \mathrm{ml}$ a $130.1 \pm 64.8 \mathrm{ml})$, el que fue concordante con el aumento de la FEVI (Tabla 4). Otra forma de analizar los datos de ambos grupos, es valorar el comportamiento de la FEVI en su seguimiento ( 0 , 3,6 , y 12 meses). Los pacientes que mejoraron este parámetro fueron catalogados como respondedores, aunque la respuesta fuera parcial. En la (Figura 2) se puede observar una clara tendencia a un mayor número de pacientes respondedores en el grupo tratado con BMCs versus el grupo control.

Esta mejoría en la FE del grupo trasplantado, se observó a pesar de su mayor deterioro hemodinámico, ya que la presencia de insuficiencia mitral moderada o severa, estuvo en ocho de sus pacientes, fue moderada en cinco casos y severa en tres. Opuesto a lo anterior, el grupo control tuvo sólo dos pacientes con insuficiencia mitral de grado importante, en un caso moderada y en el otro severa, diferencia que fue significativa ( $\mathrm{p}$ 0.04). Por otra parte la disfunción diastólica grado III estuvo presente en ocho de los pacientes tratados con BMCs y sólo en dos de los controles, diferencia que también fue significativa (p 0.04).

Otro punto de interés de nuestro estudio, es el hecho de que fue realizado en una población de pacientes ambulatorios, de consulta frecuente, no seleccionada por etiología específica, siendo todos ellos portadores de IC y FEVI baja. La mayor parte de los estudios en IAM seleccionan pacientes con FEVI superiores a las nuestras. Sólo estudios más recientes en IAM,31-33 post infarto tardío ${ }^{25,26} \mathrm{O}$ miocardiopatías, ${ }^{28}$ han seleccionado pacientes con FEVI más bajas.

Es importante destacar que aunque nuestra población de pacientes estudiados no fue homogénea respecto al origen de su cardiopatía, ambos grupos fueron semejantes. La etiología más frecuente fue la miocardiopatía dilatada, ocho pacientes del grupo trasplantado y cinco del grupo control. La existencia de infarto antiguo de pared anterior extenso estuvo presente en dos pacientes del grupo tratado y en tres del grupo control, y un paciente del grupo control presentó una cardiopatía isquémica crónica. Dos pacientes de cada grupo eran portadores de una cardiopatía hipertensiva en fase dilatada. Los trastornos de la conducción intraventricular y la presencia de fibrilación auricular no fueron significativamente diferentes en los dos grupos estudiados.

Por tanto, no hubo diferencias significativas entre ambos grupos, como también puede observarse respecto a los ti- 
pos de fármacos utilizados durante su seguimiento, siendo ambas poblaciones muy semejantes (Tabla 3).

La evaluación de la CF (NYHA) mejoró significativamente (p 0.001) en el grupo tratado a los 6 y 12 meses (Figura 1), hecho que fue temporalmente coincidente con la mejoría de la función ventricular izquierda. Los pacientes tratados partieron con un mayor deterioro en su CF, el que fue significativo al comparar ambos grupos $(\mathrm{p}<0.05)$. La evaluación a través de las encuestas de calidad de vida confirma lo anterior. Existió una mejoría significativa de la calidad de vida de los pacientes tratados con BMCs según lo evaluado por el cuestionario MLHFQ y de todos los parámetros evaluados por el cuestionario KCCQ ( $\mathrm{p}<0.01)$. En el grupo control se apreció una diferencia estadísticamente significativa sólo respecto al ítem de sintomatología del KCCQ $(\mathrm{p}<0.05)$, no encontrándose diferencias respecto otros parámetros de ese cuestionario, ni respecto al MLHFQ (Tabla 5).

Tampoco observamos entre ambos grupos diferencias significativas respecto a las arritmias, evaluadas a través del Holter. El test de esfuerzo no mostró diferencias respecto al tiempo de ejercicio, frecuencia cardíaca y presión sistólica máxima entre ambos grupos, no siendo de utilidad en la evaluación de la CF. Ninguno de los pacientes presentó isquemia durante el examen.

Las dos limitaciones más importantes de este trabajo fueron por una parte el número reducido de pacientes y por el otro la falta de randomización. Si bien ambos grupos de pacientes son bastante homogéneos en sus características, los resultados deben ser interpretados con precaución y corroborados con estudios aleatorizados y con un mayor número de casos.

Otra limitante a destacar es que al grupo control no se le administró placebo intracoronario, esto fue fundamentado en la necesidad de no someter a estos pacientes a un riesgo innecesario. Esto fue aprobado y acordado por el Comité de Ética de los centros participantes.

Otras limitaciones de nuestro estudio son las siguientes: si bien la ecocardiografía es de gran utilidad, no contamos con strain rate radial, lo que nos impidió un análisis deta- llado de los diferentes sectores del ventrículo izquierdo. Esperábamos que la RMN de corazón resolviera esta limitación, lamentablemente esta no fue de ayuda en este estudio, ya que no pudo realizarse en todos los pacientes. No se efectuó en el grupo control en tres pacientes, el primero por ser portador de un cardiodesfibrilador, el segundo por obesidad mórbida y el tercero por claustrofobia. Adicionalmente, hubo dos pacientes fallecidos tempranamente, lo que restó aún más el un número de casos para un adecuado análisis de los datos. Similarmente, los potenciales ventriculares tardíos y la variabilidad de frecuencia cardíaca no pudieron ser realizadas en todos los pacientes. La explicación de este hecho es que los potenciales ventriculares tardíos carecen de valor en presencia de fibrilación auricular y alteraciones de la conducción intraventricular, por lo que al estar presente esta condición clínica, se excluyeron del examen un número significativo de casos. Por razones semejantes, la variabilidad de la frecuencia cardíaca tampoco pudo ser efectuada en todos los pacientes, ya que fueron excluidos los casos de fibrilación auricular, marcapaso y extrasístoles frecuentes.

A pesar de las limitaciones antes señaladas, nuestro estudio nos permite concluir, al igual que otros autores, que el trasplante intracoronario con BMCs es seguro, documentando una mejoría de la FEVI a partir de los seis meses, que se mantiene en el seguimiento de los doce meses.

Es necesario considerar, que la terapia regenerativa es adicional a la terapia estándar de la IC, quedando en la actualidad muchas dudas por resolver. Entre ellas vale la pena destacar la selección de pacientes proclives a esta nueva modalidad de tratamiento, fuente celular y célula más adecuada, vías de implante, número de células a implantar y momento de su aplicación.

\section{Agradecimientos}

Servicios de Cardiología de los Hospitales: San Juan de Dios, Barros Luco Trudeau y Clínica Santa María.

Laboratorio de Terapia Celular de la Universidad de los Andes. Clínica Santa María. INNOVA Chile, CORFO. 


\section{Referencias:}

1. GJARSA JJ, KLONER RA. Left Ventricular Remodeling in the Post-Infarction Heart: a Review of Cellular Mechanisms, and Therapeutic Modalities. Heart Fail Rev 2011;16:13-21.

2. GERSH BJ, SIMARI R, BEHFAR A, TERZIC C, TERZIC A. Cardiac Cell Repair Therapy: A Clinical Perspective. Mayo Clin Proc 2009;84:876-892.

3. BOSCH X, ALFONSO F, BERMEJO J. Avancen en la Evaluación Diagnóstica y Terapéutica de la Insuficiencia Cardiaca. Del tratamiento Tónico-Depletivo al Trasplante Celular y la Regeneración Miocárdica. Rev Esp Cardiol 2004;57:161-2.

4. STEM CELL Treatment for Acute Myocardial Infarction (Review) Copyright (C) 2012. The Cochrane Collaboration. Published by John Wiley \& Sons, Ltd.

5. JEEVANANTHAM V,BUTLER M, SAADA,ABDEL-LATIF A, ZUBA-SURMA EK, DAWN B. Adult Bone Marrow Cell Therapy Improves Survival and Induces Long-Term Improvement in Cardiac Parameters: A systematic Review and MetaAnalysis. Circulation. 2012;126: 551-568.

6. ICHIM TE, SOLANO F, BRENES R, GLENN E, CHAN K, et al. Placental Mesenchymal and Cord Blood Stem Cell Therapy for Dilated Cardiomyopathy. Reproductive-BioMedicine On Line. 2008;16:898-905.

7. HARE JM, TRAVERSE JH, HENRY TD, DIB N, STRUMPF RK, SCHULMAN SP, et al. A Randomized, Double-Blind, Placebo-Controlled, Dose-Escalation Study of Intravenous Adult Human Mesenchymal Stem Cells (Prochymal) After Acute Myocardial Infarction. J Am Coll Cardiol 2009; 54: 2277- 86.

8. SOEKI T, TAMURA Y, SHINOHARA H, BANDO K, FUKUDA N. Role of Circulating Vascular Endothelial Growth Factor and Hepatocyte Growth Factor in Patients with Coronary Artery Disease. 2000; 15: 105-111.

9. ORLIC D, KAJSTURA J, CHIMENTI S, JAKONIUK I, ANDERSON SM, LI B, et al. Bone marrow cells regenerate infarcted myocardium. 2001; 410: 701-705.

10. SHINTANI S, MUROHARAT, IKEDA H, UENO T, HONMA T, KATOH A, et al. Mobilization of Endothelial Progenitor Cells in Patients with Acute Myocardial Infarction. Circulation 2001; 103: 2776-2779.

11. BELTRAMI AP, BARLUCCHI L, TORELLA D, BAKER M, LIMANA F, CHIMENTI S, et al. Adult Cardiac Stem Cells Are Multipotent and Support Myocardial Regeneration. 2003; 114: 763 -776.
12. KAJSTURA J, ROTA M, WHANG B, CASCAPERA S, HOSODA T, BEARZI C, et al. Bone Marrow Cells Differentiate in Cardiac Cell Lineages after Infarction Independently of Cell Fusion. Circ Res 2005; 96:127-137.

13. ANVERSA P, LERI A, ROTA M, HOSODA T, BEARZI C, URBANEK K, et al. Concise Review: Stem Cells, Myocardial Regeneration, and Methodological, Stem Cells 2007:25;589-601.

14. NAGAYA N, KANGAWA K, ITOH T, MURAKAMI S, MATSUMOTO T, KANGAWA K,et al. Transplantation of Mesenchymal Stem Cells Improves Cardiac Function in a Rat Model of Dilated Cardiomyopathy. Circulation 2005; 112: 1128-1135.

15. STRAUER BE, BREHM M, ZEUS T, GATTERMANN N, HERNÁNDEZ A, SORG RV, et al. Intracoronary, Human Autologous Stem Cell Transplantation for Myocardial Regeneration Following Myocardial Infarction. Dtsch Med Wochenschr 2001; 126: $932-8$

16. ASSMUS B, SCHACHINGER V, TEUPE C, et al. Transplantation of Progenitor Cells and Regeneration Enhancement in Acute Myocardial Infarction (TOPCARE-AMI). Circulation 2002. 106:3009-17.

17. STRAUER BE, BREHM BM, ZEUS T, KOESTERING M, HERNANDEZ A, SORG RV, et al. Repair of Infarcted Myocardium by Autologous Intracoronary Mononuclear Bone Marrow Cell Transplantation in Human. Circulation 2002; 106:1913-1918.

18. SCHÄCHINGER V,ERBS S, ELSÄSSER A, HABERBOSCH W, HAMBRECHT R, HÖLSCHERMANN H, et al. Improved Clinical Outcome After Intracoronary Administration of BoneMarrow-Derived Progenitor Cells in Acute Myocardial Infarction: Final 1-year Results of the REPAIR-AMI trial. Eur Heart J 2006; 27:2775-83.

19. SCHÄCHINGER V,ERBS S, ELSÄSSER A, HABERBOSCH W, HAMBRECHT R, HÖLSCHERMANN H, et al. Intracoronary Bone Marrow Derived Progenitor Cells in Acute Myocardial Infarction. N Engl J Med 2006; 355:1210-21

20. ABDEL-LATIF A, BOLLI R, TLEYJEH IM, MONTORI VM, PERIN EC, HORNUNG CA, et al. Adult Bone Marrow-Derived Cells for Cardiac Repair: A Systematic Review And MetaAnalysis. Arch Intern Med 2007; 167: 989-97.

21. YOUSEF M, SCHANNWELL CM, KÖSTERING M, ZEUS T, BREHM M, STRAUER BE. The BALANCE Study: Clinical Benefit And Long-Term Outcome After Intracoronary Autologous Bone Marrow Cell Transplantation in Patients with Acute Myocardial Infarction. J Am Coll Cardiol 2009; 53: 2262-2269. 
22. REVILLA A, LÓPEZ J, ARNOLD R, SÁNCHEZ PL, VILLA A, PINEDO M, et al. Evolución a largo plazo de la Función Ventricular tras la Terapia Celular Intracoronaria en el Infarto Agudo de Miocardio. Rev. Esp Cardio. 2011; 64: 334-337.

23. PERIN EC, DOHMANN HF, BOROJEVIC R, SILVA SA, SOUSA AL, MESQUITA CT, et al. Transendocardial, Autologous Bone Marrow Cell Transplantation for Severe, Chronic Ischemic Heart Failure. Circulation 2003;107: r75-r83.

24. STAMM C, KLEINE HD, CHOI YH, DUNKELMANN S, LAUFFS JA, LORENZEN B, et al. Intramyocardial Delivery of CD133 Bone Marrow Cells and Coronary Artery Bypass Grafting for Chronic Ischemic Heart Disease: Safety and Efficacy Studies. The Journal of Thoracic and Cardiovacular Surgery 2007;133: 3. 717-725.e5.

25. STRAUER BE, YOUSEF M, SCHANNWELL CM. The Acute and Long-Term Effects of Intracoronary Stem Cell Trasplantation in 191 Patiens with Chronic Heart Failure: The Star-Heart Study. European Journal of Heart Failure. 2010; 12: 721-729.

26. WILLIAMS AR, TRACHTENBERG B, VELAZQUEZ DL, MCNIECE I, ALTMAN P, ROUY D, et al. Hare Intramyocardial Stem Cell Injection in Patients with Ischemic: Cardiomyopathy Functional Recovery and Reverse Remodeling Circ Res. 2011;108:792-796.

27. VRTOVEC B, POGLAJEN G, SEVER M, LEZAIC L, DOMANOVIC D, CERNELC P, et al. Effects of Intracoronary Stem Cell Transplantation in Patients with Dilated Cardiomyopathy. Journal of Cardiac Failure 2011; 17: 272-281.

28. VRTOVEC B, POGLAJEN G, LEZAIC L, SEVER M, DOMANOVIC D, CERNELC P, et al. Efeccts of Intracoronary CD34+ Stem Cell Transplantation in Non-Ischemic Dilated Cardiomyopathy Patients: 5-Year Follow. Circ Res AHA. 2013; 112: $165-73$

29. GRAJECK S, POPIEL M, GIL L, BREBOROWICZ P, LESIAK M, CZEPCZYNSKI R, et al. Influence of Bone Marrow Stem Cells on Left Ventricle Perfusion and Ejection Fraction in Patients with Acute Myocardial Infarction of Anterior Wall: Randomized Clinical Trial: Impact of Bone Marrow Stem Cell Intracoronary Infusion on Improvement of Microcirculation. Eur Heart J. 2010;31:691-702.

30. ASSMUS B, ROLF A, ERBS S, ELSASSER A, HABERBOSCH W, HAMBRECHT R, et al. REPAIR-AMI Investigators. Clinical Outcome 2 Years After Intracoronary Administration of Bone Marrow-Derived Progenitor Cells in Acute Myocardial Infarction. Circ Heart Fail. 2010;3:89-96.

31. TENDERA M, WOJAKOWSKI W, RUZYLLO W, CHOJ-
NOWSKA L, KEPKA C, TRACZ W, et al. Intracoronary Infusion of Bone Marrow-Derived Selected CD34+ CXCR4+ Cells and Non-Selected Mononuclear Cells in Patients with Acute STEMI and Reduced Left Ventricular Ejection Fraction: Results of Randomized, Multicenter Myocardial Regeneration by Intracoronary Infusion of Selected Population of Stem Cells in Acute Myocardial Infarction (REGENT) Trial. Eur Heart J. 2009. 30, 1313-1321.

32. HIRSCH A, NIJVELDT R, VAN DER VLEUTEN PA, TIJSSEN JG, VAN DER GIESSEN WJ, TIO RA, et al. Intracoronary Infusión of Mononuclear Cells from Bone Marrow or Perpheral Blood Compared with Standart Therapy in Patients After Acute Myocardial Infarction Treated by Primary Percutaneous Coronary Intervention: Results of the Randomized Controlled HEBE Trial. Eur Heart J. 2011; 32: 1736-1747.

33. RONCALLI J, MOUQUET F, PIOT C, TROCHU JN, LE CORVOISIER P, NEUDER Y, et al. Intracoronary Autologous Mononucleated Bone Marrow Cell Infusion for Acute Myocardial Infarction: Results of the Randomized Multicenter BONAMI Trial. Eur Heart J. 2011; 32: 1748-1757.

30. ASSMUS B, ROLF A, ERBS S, ELSASSER A, HABERBOSCH W, HAMBRECHT R, TILLMANNS H, YU J, et al. REPAIR-AMI Investigators. Clinical Outcome 2 Years After Intracoronary Administration of Bone Marrow-Derived Progenitor Cells in Acute Myocardial Infarction. Circ Heart Fail. 2010;3:89-96.

31. TENDERA M, WOJAKOWSKI W, RUZUTTO W, et al. Intracoronary Infusion of Bone Marrow-Derived Selected CD34+ CXCR4+ Cells and Non-Selected Mononuclear Cells in Patients with Acute STEMI and Reduced Left Ventricular Ejection Fraction: Results of Randomized, Multicenter Myocardial Regeneration by Intracoronary Infusion of Selected Population of Stem Cells in Acute Myocardial Infarction (REGENT) Trial. Eur Heart J. 2009. 30, 1313-1321.

32. HIRSCH A, NIJVELDT R, VAN DER VLEUTEN P, et al. Intracoronary Infusión of Mononuclear Cells from Bone Marrow or Perpheral Blood Compared with Standar Therapy in Patients After Acute Myocardial Infarction Treated by Primary Percutaneous Coronary Intervention: Results of the Randomized Controlled HEBE Trial. Eur Heart J. 2011. 32, 1736-1747.

33. JONCALLI J, MOUQUET F, PIOT C, et al. Intracoronary Autologous Mononucleated Bone Marrow Cell Infusion for Acute Myocardial Infarction: Results of the Randomized Multicenter BONAMI Trial. Eur Heart J. 2011. 32, 1748-1757. 